\title{
A five dimensional model of varying effective gravitational and fine structure constants
}

\begin{abstract}
J. P. Mbelek and M. Lachièze-Rey
Service d'Astrophysique, C.E. Saclay, 91191 Gif-sur-Yvette Cedex, France

Received 23 May 2002 / Accepted 7 October 2002

Abstract. We explore the possibility that the reported time variation of the fine structure constant $\alpha$ is due to a coupling between electromagnetism and gravitation. We predict such a coupling from a very simple effective theory of physical interactions, under the form of an improved version of the Kaluza-Klein theory. We show that it precisely leads to a variation of the effective fine structure constant with cosmic conditions, and thus with cosmic time. The comparison with the recent data from distant quasars absorption line spectra gives a good agreement; moreover, this may reconcile the claimed results on $\alpha$ with the upper limit from the Oklo naturel Uranium fission reactor.
\end{abstract}

Key words. galaxies: quasars: absorption lines - cosmology: theory

\section{Introduction}

Recent publications (Webb et al. 2001; Murphy et al. 2001) report observations of distant quasars absorption lines, which may indicate a time variation of the fine structure constant $\alpha$. Different kinds of explications have been proposed (see in conclusion), which all involve new physics (Barrow et al. 2002a; Barrow et al. 2002b; Sandvik et al. 2002; Dvali \& Zaldarriaga 2002; Ahluwalia \& Kirchbach 2001; Olive \& Pospelov 2001). Since many theoretical arguments suggest that our present theories of physical interactions are not the ultimate ones, this possibility deserves serious attention.

An ultimate theory would include additional fields and coupling which remain presently unknown. At the effective level, and in particular in astrophysical conditions, those can be manifest as a soft dependence of the "constants" of the interactions with some parameters. For instance, the effective theory considered here predicts a variation of the effective gravitational constant $G_{\text {eff }}$ with respect to electromagnetic conditions, and a variation of the effective $\alpha$ with respect to gravitational conditions, and thus with the cosmic time (seen as a parameter expressing the variation of the cosmic gravitational potential, through the Friedmann-Lemaitre equations). In this paper, we calculate the expected variation of $\alpha$, and compare it with astronomical observations. In a companion paper (Paper II), we have shown that the predicted dependence of $G_{\text {eff }}$ with the value of the geomagnetic field, in the same framework, may explain the discordant terrestrial measurements of the gravitational constant.

One of the simplest effective theories that it is possible to build (beside Brans-Dicke type theories) results from the compactification of the Kaluza-Klein (KK) one. As we show here (see also Lichnerowicz 1955), this leads to replace the gravitational constant $G$ and the fine structure constant $\alpha$ by effective

Send offprint requests to: J. P. Mbelek, e-mail: mbelek@discovery.saclay.cea.fr values, which vary with the scalar field $\Phi$ introduced in the theory. On the other hand, different authors (Salam \& Strathdee 1982; Witten 1982) have pointed out that a pure KK action leads to instability of the theory, because of the bad sign for the kinetic term of $\Phi$ (Sokolowski \& Golda 1987). The same authors suggested the presence of an additional field to cure this problem. In a previous study (Paper I), we applied an argument initially from Landau \& Lifshitz (1959), to study this instability, and we proposed a minimal hypothesis for stabilization: the addition of an external bulk scalar field $\psi$. Thus we consider this modified KK theory (hereafter $\mathrm{KK} \psi$ ) as the simplest candidates for an effective theory, a prototype to explore the possibility that the observational results are due to a coupling between gravitation and electromagnetism (hereafter GE coupling).

Seen in our 4-dimensional space-time, the $\mathrm{KK} \psi$ Lagrangian leads to a theory of gravitation and electromagnetism, with the additional fields $\psi$ (external stabilizing field) and $\Phi$ (internal KK field). The latter induce a GE coupling, which appears precisely as a dependence of the (effective) constants $G_{\text {eff }}$ and $\alpha_{\text {eff }}$ with respect to other fields. This paper explores the cosmic evolution of $\alpha_{\text {eff }}$ generated by that of matter and gravitation (spacetime curvature).

In Sect. 2, we calculate the variations predicted by the $\mathrm{KK} \psi$ theory: Sect. 2.1 recalls the definition of the effective coupling constants, and gives the effective Maxwell-Einstein equations in the context of the compactified KK theory; Sect. 2.2 introduces the $\mathrm{KK} \psi$ Lagrangian and the resulting equations (Maxwell-Einstein and scalar field evolutions); Sect. 2.3 considers the cosmological solutions. In Sect. 3, we compare our calculations of the cosmological evolution of the fine structure constant with the available data from distant quasars absorption lines. Also, we discuss the consistency of our results especially with respect to the Oklo phenomenon. In Sect. 4, the similarities and differences with other work are emphasized. 


\section{Effective coupling constants}

\subsection{The Kaluza-Klein theory}

The original KK theory, after dimensional reduction, leads to the effective action in the Jordan-Fierz frame (e.g., see Thiry 1948; Lichnerowicz 1955)

$$
\begin{aligned}
S_{\mathrm{KK}, 4}= & -\int \sqrt{-g}\left[\frac{c^{4}}{16 \pi} \frac{\Phi}{G} R+\frac{1}{4} \varepsilon_{0} \Phi^{3} F_{\alpha \beta} F^{\alpha \beta}\right. \\
& \left.+\frac{c^{4}}{4 \pi G} \frac{\partial_{\alpha} \Phi \partial^{\alpha} \Phi}{\Phi}\right] \mathrm{d}^{4} x,
\end{aligned}
$$

where $A^{\alpha}$ is the potential 4-vector of the electromagnetic field, $F_{\alpha \beta}=\partial_{\alpha} A_{\beta}-\partial_{\beta} A_{\alpha}$ is the electromagnetic field strength tensor, $\Phi$ the (internal) scalar field related by $\hat{g}_{44}=-\Phi^{2}$ to the fifteenth degree of freedom, $\hat{g}_{44}$, of the 5-dimensional metric and $G$ the (true) gravitational constant. We emphasize that this is not a theory with minimally coupled scalar field. According to Lichnerowicz (1955), the quantity $G_{\text {eff }}:=\frac{G}{\Phi}$ of the EinsteinHilbert term, and the factor $\varepsilon_{0 \text { eff }}=\varepsilon_{0} \Phi^{3}$ of the Maxwell term in (1), should be interpreted respectively as the effective gravitational "constant" and the effective vacuum dielectric permittivity. These terms depend on the local (for terrestrial experiments) or global (at cosmological scale) value of $\Phi$, assumed to be positive defined. Clearly, the previous considerations lead to an effective fine structure constant

$\alpha_{\text {eff }}=e^{2} / 4 \pi \varepsilon_{0 \mathrm{eff}} \hbar c=\frac{\alpha}{\Phi^{3}}$,

to be compared to the true fine structure constant $\alpha$ := $e^{2} / 4 \pi \varepsilon_{0} \hbar c$. It is worth noticing that this does not involve any variation of the electric charge, unlike the earlier suggestion of Bekenstein (1982). Also, the velocity of light remains constant since the value of the effective vacuum magnetic permeability, $\mu_{0 \mathrm{eff}}=\mu_{0} \Phi^{-3}$, insures $\varepsilon_{0 \mathrm{eff}} \mu_{0 \mathrm{eff}}=\varepsilon_{0} \mu_{0}$ (see Lichnerowicz 1955). Applying the least action principle to the action (1) yields

- the generalized Einstein-Maxwell equations with the additional source term

$T_{\alpha \beta}^{(\Phi)}=\frac{c^{4}}{8 \pi G}\left(\nabla_{\alpha} \nabla_{\beta} \Phi-g_{\alpha \beta} \nabla_{v} \nabla^{v} \Phi\right)$,

in addition to the electromagnetic stress-energy tensor $T_{\alpha \beta}^{(\mathrm{EM})}$. They identify to the usual expressions, where $G$ and $\varepsilon_{0}$ are replaced respectively by $G_{\text {eff }}$ and $\varepsilon_{0 \mathrm{eff}}$.

- the KK scalar field equation

$$
\nabla_{v} \nabla^{v} \Phi=-\frac{4 \pi G}{c^{4}} \varepsilon_{0} \Phi^{3} F_{\alpha \beta} F^{\alpha \beta}
$$

\subsection{Stabilizing the Kaluza-Klein action}

To stabilize the KK action (Paper I), the simplest possibility is to introduce an additional matter field: a real bulk scalar field minimally coupled to gravity. After dimensional reduction, this field appears as a scalar field $\psi$ in spacetime, with the effective action (in the Jordan-Fierz frame)

$$
\begin{aligned}
S_{4}= & S_{\mathrm{KK}, 4}+S_{\psi, 4}=S_{\mathrm{KK}, 4} \\
& +\frac{c^{4}}{4 \pi G} \int \sqrt{-g} \Phi\left[\frac{1}{2} \partial_{\alpha} \psi \partial^{\alpha} \psi-U-J \psi\right] \mathrm{d}^{4} x,
\end{aligned}
$$

where $U=U(\psi)$ denotes the self-interaction potential of $\psi$ and $J$ its source term. The latter includes contributions from the matter and from $\Phi$, both proportional to the trace of their energy-momentum tensor, viz. $\frac{8 \pi G}{3 c^{4}} g(\psi, \Phi) T_{\alpha}^{\alpha}$, and that of the (traceless) electromagnetic field, $\varepsilon_{0} f(\psi, \Phi) F_{\alpha \beta} F^{\alpha \beta}$. Generally speaking these coupling functions are temperature dependent, with magnitude decreasing as the temperature increases (this prevents from any significant modification of the big bang nucleosynthesis). The necessity to recover the usual physics whenever the scalar fields are not excited requires $g(v, 1)=f(v, 1)=0$ and $U(v)=0$. We have written $v$ the vacuum expectation value $(\mathrm{VEV})$ of $\psi$, such that $\frac{\partial U}{\partial \psi}(v)=0$ (definition of the VEV of $\psi$ ). The definition of $G_{\text {eff }}$ implies that the VEV of $\Phi$ is 1 .

Applying the variational principle to (5) yields

$\nabla_{v} \nabla^{v} \psi=-J-\frac{\partial J}{\partial \psi} \psi-\frac{\partial U}{\partial \psi}$

and

$$
\begin{aligned}
\nabla_{v} \nabla^{v} \Phi= & -\frac{4 \pi G}{c^{4}} \varepsilon_{0} F_{\alpha \beta} F^{\alpha \beta} \Phi^{3}+U \Phi+J \psi \Phi \\
& +\frac{\partial J}{\partial \Phi} \Phi^{2} \psi-\frac{1}{2}\left(\partial_{\alpha} \psi \partial^{\alpha} \psi\right) \Phi
\end{aligned}
$$

The effective Einstein equations are unchanged, apart from a new source term: the effective energy momentum tensor of $\psi$,

$T_{\alpha \beta}^{(\psi)}=\frac{c^{4}}{4 \pi G_{\mathrm{eff}}}\left[\partial_{\alpha} \psi \partial_{\beta} \psi-\left(\frac{1}{2} \partial_{\gamma} \psi \partial^{\gamma} \psi-U-J \psi\right) g_{\alpha \beta}\right]$.

Since we know that, for the effects examined here, the effective values are close to the usual one, we linearize the two scalar fields around their respective VEVs. Hence, Eqs. (6), (7) above reduce to

$\nabla_{v} \nabla^{v} \psi=-\frac{\partial J}{\partial \psi} v$

and

$\nabla_{v} \nabla^{v} \Phi=-\frac{4 \pi G}{c^{4}} \varepsilon_{0} F_{\alpha \beta} F^{\alpha \beta}+\frac{\partial J}{\partial \Phi} v-\frac{1}{2} \partial_{\alpha} \psi \partial^{\alpha} \psi$

\subsection{Cosmological solutions}

For cosmology, we assume spatially constant values of the fields and we follow their evolutions with respect to the cosmic time, $t$. Hence, $\psi=\psi(t)$ and $\Phi=\Phi(t)$. Besides, $F_{\alpha \beta} F^{\alpha \beta}$ vanishes for the pure EM cosmic background radiation. Thus, the cosmological equations reduce to the effective Friedmann equation (with a cosmological constant $\Lambda$ )

$\left(\frac{\dot{a}}{a}\right)^{2}=\frac{8 \pi G}{3} \rho-\frac{k c^{2}}{a^{2}}+\frac{\Lambda c^{2}}{3}+\frac{1}{3} \dot{\psi}^{2}-\frac{1}{6}(\ddot{\Phi}+6 H \dot{\Phi})$,

$\frac{\ddot{a}}{a}=-\frac{4 \pi G}{3}\left(\rho+3 \frac{P}{c^{2}}\right)+\frac{\Lambda c^{2}}{3}-\frac{1}{3} \dot{\psi}^{2}-\frac{1}{6}(\ddot{\Phi}+3 H \dot{\Phi})$ 
and, for the scalar fields,

$\ddot{\psi}+3 H \dot{\psi}=-\frac{8 \pi G}{3} \beta_{\psi} v\left(\rho-3 \frac{P}{c^{2}}\right)$

$\ddot{\Phi}+3 H \dot{\Phi}=-\frac{1}{2} \dot{\psi}^{2}+\frac{8 \pi G}{3} \beta_{\Phi} v\left(\rho-3 \frac{P}{c^{2}}\right)$.

The dot denotes the derivative with respect to the cosmic time, $H=\dot{a} / a$ is the expansion rate (Hubble parameter), $a=a(t)$ the scale factor, $k$ is the spatial curvature parameter and $P$ the pressure; we have set $\beta_{\psi}=\frac{\partial g}{\partial \psi}(v, 1)$ and $\beta_{\Phi}=\frac{\partial g}{\partial \Phi}(v, 1)$. The smallness of the observed effects implies $\left|\beta_{\Phi} v\right| \ll 1,\left|\beta_{\psi} v\right| \ll 1$, whereas the consistency of the model implies $|\dot{\psi}| \ll H$ and $|\dot{\Phi}| \ll H$ (all confirmed by the numerical calculations below). Hence, the small excitations of the scalar fields do not modify significantly the variation of the scale factor with respect to the cosmic time. As this is suggested by observations, we assume zero spatial curvature. Let us emphasize that Eq. (14) implies that the extrema of $\Phi$ are necessary maxima during the matter or matter- $\Lambda$ dominated era (present era). On account of Eq. (13), the same conclusion applies to $\psi$ under the condition $\beta_{\psi} v>0$ (choosing $\beta_{\psi} v<0$ would lead to minima of $\psi$, instead).

\subsubsection{Radiation era}

Before the recombination, the content of the universe is well described by the equation of state $P=\frac{1}{3} \rho c^{2}$ (matter negligible, no spatial curvature): putting $H=1 / 2 t, a=a\left(t_{0}\right)\left(t / t_{0}\right)^{1 / 2}, \rho=$ $\rho\left(t_{0}\right)\left(t_{0} / t\right)^{2}$, we obtain

$\ddot{\psi}+\frac{3}{2 t} \dot{\psi}=0$,

$\ddot{\Phi}+\frac{3}{2 t} \dot{\Phi}=-\frac{1}{2} \dot{\psi}^{2}$,

where $t_{0}$ is the present time. The solutions of Eqs. (15) and (16) take the forms

$\psi=v+\delta \psi\left(t_{\mathrm{d}}\right)\left(\frac{t_{\mathrm{d}}}{t}\right)^{1 / 2}$

and

$\Phi=1+\frac{1}{4} \delta \psi\left(t_{\mathrm{d}}\right)^{2} \frac{t_{\mathrm{d}}}{t}+\left[\delta \Phi\left(t_{\mathrm{d}}\right)-\frac{1}{4} \delta \psi\left(t_{\mathrm{d}}\right)^{2}\right]\left(\frac{t_{\mathrm{d}}}{t}\right)^{1 / 2}$,

where $\delta \psi\left(t_{\mathrm{d}}\right)=\psi\left(t_{\mathrm{d}}\right)-v, \delta \Phi\left(t_{\mathrm{d}}\right)=\Phi\left(t_{\mathrm{d}}\right)-1$ and $t_{\mathrm{d}}$ denotes the epoch of matter-radiation decoupling. Now, requiring that both $\psi$ and $\Phi$ be bounded at any time past the big bang involves $\delta \psi\left(t_{\mathrm{d}}\right)=0$ and $\delta \Phi\left(t_{\mathrm{d}}\right)=0$. Hence, both scalar fields remain constant and equal to their respective VEV, during the radiation era. As a consequence, the effective fine structure constant identifies to the true fine structure constant during the radiation era.

\subsubsection{Model without cosmological constant}

After recombination, the matter and the cosmological constant play their role. We explore two different cosmological models: first, in this section, an Einstein-de Sitter model, with no cosmological constant and the critical density (no spatial curvature, no pressure). In the next section we explore a more realistic model with a cosmological constant. Putting $H=2 / 3 t$, $a=a\left(t_{0}\right)\left(t / t_{0}\right)^{2 / 3}, \rho=\rho\left(t_{0}\right)\left(t_{0} / t\right)^{2}$, we obtain

$\ddot{\psi}+\frac{2}{t} \dot{\psi}=-\frac{4}{9} \frac{\beta_{\psi} v}{t^{2}}$

$\ddot{\Phi}+\frac{2}{t} \dot{\Phi}=-\frac{1}{2} \dot{\psi}^{2}+\frac{4}{9} \frac{\beta_{\Phi} v}{t^{2}}$.

The solutions of Eqs. (19) and (20) take the forms

$\psi=v+\delta \psi_{0} \frac{t_{0}}{t}-\frac{4}{9} \beta_{\psi} v \ln \left(\frac{t}{t_{0}}\right)$

and

$$
\begin{aligned}
\Phi= & 1+\left(\delta \Phi_{0}+\frac{1}{4} \delta \psi_{0}^{2}\right) \frac{t_{0}}{t}-\frac{1}{4} \delta \psi_{0}^{2}\left(\frac{t_{0}}{t}\right)^{2} \\
& +\frac{4}{9} v\left[\beta_{\Phi}+\beta_{\psi} \delta \psi_{0} \frac{t_{0}}{t}\right] \ln \left(\frac{t}{t_{0}}\right),
\end{aligned}
$$

where $t_{0}$ denotes the present epoch in the cosmic time, and we have set $\delta \psi_{0}=\psi\left(t_{0}\right)-v$ and $\delta \Phi_{0}=\Phi\left(t_{0}\right)-1$. On account of the results obtained previously at the epoch of matter-radiation decoupling, one gets in addition the following constraints

$\delta \psi_{0}=-\frac{4}{9} \beta_{\psi} v \frac{t_{\mathrm{d}}}{t_{0}} \ln \left(\frac{t_{0}}{t_{\mathrm{d}}}\right)$

and

$\delta \Phi_{0}=\frac{1}{4} \delta \psi_{0}^{2} \frac{t_{0}-t_{\mathrm{d}}}{t_{\mathrm{d}}}+\frac{4}{9} v\left[\beta_{\Phi} \frac{t_{\mathrm{d}}}{t_{0}}+\beta_{\psi} \delta \psi_{0}\right] \ln \left(\frac{t_{0}}{t_{\mathrm{d}}}\right)$.

\subsubsection{Model with cosmological constant}

\section{Present era}

The present cosmological data seem to favor a model where $\Omega_{\Lambda}=2 \Omega_{\mathrm{m}} \approx 2 / 3$, that we explore now (still no spatial curvature and no pressure). We write the solution as $H=H_{0} \sqrt{\lambda} \operatorname{coth} x, a=a\left(t_{0}\right)\left(\Omega_{\mathrm{m}} / \lambda\right)^{1 / 3}[\sinh x]^{2 / 3}, \rho=$ $\rho\left(t_{0}\right)\left[a\left(t_{0}\right) / a(t)\right]^{3}$ where $\lambda=\Omega_{\Lambda}=\Lambda c^{2} / 3 H_{0}^{2}$, and $x=$ $\frac{3}{2} \sqrt{\lambda} H_{0} t$. Solving Eqs. (13) and (14), we obtain

$$
\begin{aligned}
\psi= & v+\frac{2}{9} \beta_{\psi} v \ln \lambda+\delta \psi_{\Lambda} \sqrt{\lambda} \operatorname{coth} x \\
& -\frac{4}{9} \beta_{\psi} v[1-x \operatorname{coth} x+\ln (\sinh x)]
\end{aligned}
$$

and

$$
\begin{aligned}
\Phi= & 1-\frac{2}{9} \beta_{\Phi} v \ln \lambda+\sqrt{\lambda}\left(\delta \Phi_{\Lambda}+\frac{4}{9} \beta_{\psi} v \delta \psi_{\Lambda} \sqrt{\lambda}\right. \\
& \left.+\frac{1}{4} \delta \psi_{\Lambda}^{2}\right) \operatorname{coth} x+\frac{4}{9} \beta_{\Phi} v[1-x \operatorname{coth} x+\ln (\sinh x)] \\
& -\frac{1}{4} \delta \psi_{\Lambda}^{2} \lambda \sinh ^{-2} x-\frac{2}{9} \beta_{\psi} v \delta \psi_{\Lambda} \sqrt{\lambda}\left[\frac{3}{2} x\right. \\
& +\frac{1}{2} x \sinh ^{-2} x+\frac{1}{2} \operatorname{coth} x-\operatorname{coth} x \ln (\sinh x) \\
& \left.+x \sinh ^{2} x-\frac{1}{4} \sinh 2 x-\frac{1+\ln (\sinh x)}{\sinh x}\right]
\end{aligned}
$$


The parameters $\delta \psi_{\Lambda}$ and $\delta \Phi_{\Lambda}$ have been introduced in such a way that, formally, they reduce respectively to $\delta \psi_{0}=$ $\psi\left(t_{0}\right)-v$ and $\delta \Phi_{0}=\Phi\left(t_{0}\right)-1$ for $\Lambda=0$. Further, because of the matching conditions at $t_{\mathrm{d}}$, relations (25) and (26) are respectively subject to the constraints

$$
\begin{aligned}
\delta \psi_{\Lambda}= & \frac{1}{\sqrt{\lambda}} \frac{4}{9} \beta_{\psi} v\left[1-\frac{1}{2} \ln \lambda-x_{\mathrm{d}} \operatorname{coth} x_{\mathrm{d}}\right. \\
& \left.+\ln \left(\sinh x_{\mathrm{d}}\right)\right] \tanh x_{\mathrm{d}}
\end{aligned}
$$

and

$$
\begin{aligned}
\delta \Phi_{\Lambda}= & -\frac{4}{9} \beta_{\psi} v \delta \psi_{\Lambda} \sqrt{\lambda}-\frac{1}{\sqrt{\lambda}} \frac{4}{9} \beta_{\Phi} v\left[1-\frac{1}{2} \ln \lambda\right. \\
& \left.-x_{\mathrm{d}} \operatorname{coth} x_{\mathrm{d}}+\ln \left(\sinh x_{\mathrm{d}}\right)\right] \tanh x_{\mathrm{d}} \\
& -\frac{1}{4} \delta \psi_{\Lambda}^{2}\left[1-2 \sqrt{\lambda} \sinh ^{-1} 2 x_{\mathrm{d}}\right]+\frac{2}{9} \beta_{\psi} v \delta \psi_{\Lambda}\left[\frac{1}{2}\right. \\
& +\frac{1}{2} x_{\mathrm{d}}\left(\tanh x_{\mathrm{d}}+\sinh 2 x_{\mathrm{d}}\right)-\ln \left(\sinh x_{\mathrm{d}}\right) \\
& \left.+\frac{x_{\mathrm{d}}}{\sinh 2 x_{\mathrm{d}}}-\frac{1}{2} \sinh ^{2} x_{\mathrm{d}}-\frac{1+\ln \left(\sinh x_{\mathrm{d}}\right)}{\cosh x_{\mathrm{d}}}\right]
\end{aligned}
$$

where we have set $x_{\mathrm{d}}=\frac{3}{2} \sqrt{\lambda} H_{0} t_{\mathrm{d}}$, that is

$x_{\mathrm{d}}=\ln \left(\sqrt{\frac{\lambda}{\Omega_{\mathrm{m}}}}\left(1+z_{\mathrm{d}}\right)^{-3 / 2}+\sqrt{1+\frac{\lambda}{\Omega_{\mathrm{m}}\left(1+z_{\mathrm{d}}\right)^{3}}}\right)$.

Clearly, because of the constraints (23), (24), (27) and (28), only two parameters (the coupling constants $\beta_{\Phi} v$ and $\beta_{\psi} v$ ) are left free to fit the data.

\section{The future universe (cosmological constant era)}

In the future of the universe, the matter density and pressure become quite negligible with respect to the $\Lambda$ term: $P=\rho=$ 0 , still no spatial curvature. Putting $a \propto \exp (-\sqrt{\Lambda / 3} \mathrm{ct})$ assuming $\Lambda>0$, we obtain

$$
\begin{aligned}
& \ddot{\psi}+c \sqrt{3 \Lambda} \dot{\psi}=0, \\
& \ddot{\Phi}+c \sqrt{3 \Lambda} \dot{\Phi}=-\frac{1}{2} \dot{\psi}^{2} .
\end{aligned}
$$

The solutions of Eqs. (30) and (31) are respectively of the same form as (17) and (18). Hence, each scalar field tends to a constant equal to its VEV, during the cosmological constant era. As a consequence, here again the effective fine structure constant will approach asymptotically the true fine structure constant.

\section{Comparison with the observational data}

We compare our prediction (2) of the time variation of $\alpha_{\text {eff }}$, for the two different cosmological models, with the observational data. A quasar of redshift $z=\frac{a\left(t_{0}\right)}{a\left(t_{\mathrm{e}}\right)}-1$ emits photons at $t_{\mathrm{e}}$, that we receive at $t_{0}$ on Earth. Defining $\alpha_{z}=\alpha_{\text {eff }}\left(t_{\mathrm{e}}\right), \alpha_{0}=\alpha_{\text {eff }}\left(t_{0}\right)$

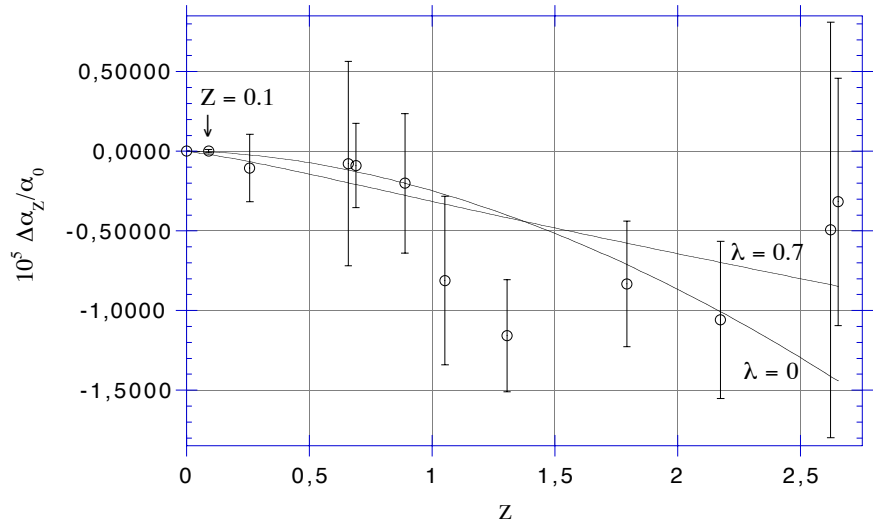

Fig. 1. Observed data and predicted curve $\Delta \alpha_{z} / \alpha_{0}$ versus the redshift. The fits correspond respectively to $\chi^{2}=0.948(\lambda=0)$ and $\chi^{2}=0.779$ $(\lambda=0.7)$ per d.o.f., which seems to favor the late time $\lambda$ dominated cosmology. Including the Oklo bounds $(z=0.1)$ in the data set would yield respectively $\chi^{2}=0.882(\lambda=0)$ and $\chi^{2}=1.094(\lambda=0.7)$ per d.o.f.

and $\Delta \alpha_{z}=\alpha_{z}-\alpha_{0}$, a least-squares fit to the observational data (Fig. 1 of Webb et al. 2001) gives,

- for $\lambda=0: \beta_{\Phi} v \simeq 8.014 \times 10^{-7}$ and $\left|\beta_{\psi} v\right| \simeq 0.0793$, with $\chi^{2}=0.948$ per degree of freedom (d.o.f.).

- for $\lambda=0.7: \beta_{\Phi} v \simeq 3.393 \times 10^{-6}$ and $\left|\beta_{\psi} v\right| \simeq 0.0317$, with $\chi^{2}=0.779$ per d.o.f.

Figure 1 shows, in the same plot, the observational results (Webb et al. 2001) and our theoretical prediction (2) of $\Delta \alpha_{z} / \alpha_{0}$ versus the redshift, for our two models $\lambda=0$ and $\lambda=0.7$ (assuming no spatial curvature).

The consistency of the recent observation from the distant quasars absorption line spectra with the constraints from the Oklo uranium deposit have been discussed by Webb et al. (2001). At the corresponding redshift, our best fits imply (assuming a spatially flat universe):

$\left(\alpha_{\text {Oklo }}-\alpha_{0}\right) / \alpha_{0} \simeq-0.4 \times 10^{-7}$ for $\lambda=0$

$\left(\alpha_{\text {Oklo }}-\alpha_{0}\right) / \alpha_{0} \simeq-1.9 \times 10^{-7}$ for $\lambda=0.7$.

Including the Oklo point in the fit modify the $\chi^{2}$ as indicated in the figure caption, and we conclude that our two best fits are consistent with the Oklo bounds. This gives an averaged decreasing rate approximately equal to $-10^{-17}$ per year, consistent with the recent analyses (Damour \& Dyson 1996; Fujii et al. 2000) on account of the remark made by Webb et al. (2001), Fujii (2002) and Fujii et al. (2002) for the case of a non-linear time-evolution in $\Delta \alpha_{z} / \alpha_{0}$. Note that this implies also a non trivial cosmic evolution for $G_{\text {eff }}$ which yields $\dot{G}_{\text {eff }} / G_{\text {eff }} \simeq-1.6 \times 10^{-17}$ per year at present, consistent with all the current bounds.

\section{Discussion and conclusion}

In the radiation dominated era, energy is present in the form of radiation only, so that the source terms for the scalar fields (Eqs. (15) and (16)) cancel. Therefore, both $\psi$ and $\Phi$ remain close to their respective VEV's. As a consequence, the effective fine structure constant $\alpha_{\text {eff }}$ remains practically constant and close to the true fine structure constant $\alpha$. It follows that, 
both for BBN and at $z=1000$ (the epoch of matter-radiation decoupling), $\Delta \alpha_{z} / \alpha_{0} \simeq-2.5 \times 10^{-4}(\lambda=0.7)$ and $\Delta \alpha_{z} / \alpha_{0} \simeq$ $-1.5 \times 10^{-10}(\lambda=0)$, in accordance with, or much below, the present observational bound (model dependent) which gives $\left|\Delta \alpha_{z} / \alpha_{0}\right|<10^{-4}-10^{-2}$ (see Battye et al. 2001 and references therein; Avelino et al. 2001; Avelino et al. 2000).

At the onset of the matter- $\Lambda$ dominated era, the scalar fields will continuously start to vary ( $\Phi$ increases), though at a lesser extent than the density of the ordinary matter. The Hubble friction term introduces a relaxation time $\tau$ of the order $1 / 3 H$. This provides a natural and sufficient way of driving back $\alpha_{\text {eff }}$ $\left(\simeq \alpha / \Phi^{3}\right)$ to its constant value, $\alpha$, after a lapse of time of a few $\tau$. As an estimate, let us consider the Einstein-de Sitter cosmology: after $2 \tau=2 / 3 H_{0}=t_{0}$, one gets $\left|\alpha_{\text {eff }}-\alpha\right|_{\max } \simeq$ $e^{-2}\left|\alpha_{\text {eff }}-\alpha\right|_{t=t_{0}}$. Since $\alpha_{\text {eff }} \simeq \alpha$ during the radiation dominated era, it follows $\left|\Delta \alpha_{\text {eff }} / \alpha_{0}\right|_{\max } \simeq 0.135\left|\Delta \alpha_{\text {eff }} / \alpha_{0}\right|_{\text {BBN }}$. Hence, $\left|\Delta \alpha_{\text {eff }} / \alpha_{0}\right|_{\max }<1.35 \times 10^{-5}-1.35 \times 10^{-3}$ as observed, on account of the bound on the variation of the fine structure constant at BBN.

Variations of the effective weak and strong coupling constants are also expected in the higher dimensional theories candidates for unification. The properties of the fundamental interactions are connected to the topological properties of the compacted extradimensions. Such theories involve more than one extradimension in order to encompass all of the gauge groups of the standard model of particle physics. In this framework, the effective constants of the gauge fields would be expressed as functions of additional internal fields $\Phi_{1}, \ldots, \Phi_{n}$. The effective electromagnetic (fine structure constant), weak and strong coupling constants would be written, respectively, as $\alpha_{\text {eff }}=\alpha F_{1}\left(\Phi_{1}, \ldots, \Phi_{n}\right), \alpha_{\text {weff }}=\alpha_{w} F_{2}\left(\Phi_{1}, \ldots, \Phi_{n}\right)$ and $\alpha_{\text {seff }}=$ $\alpha_{s} F_{3}\left(\Phi_{1}, \ldots, \Phi_{n}\right)$. The functions $F_{1}$ and $F_{2}$ are related to each other, because of the electroweak unification; and to $F_{3}$, if an unification scheme is already present. Hence, we expect, at any given time scale (dropping the "eff" indexes for clarity): $\frac{\dot{\alpha}_{w}}{\alpha_{w}}=\frac{\partial \ln F_{2}}{\partial \ln F_{1}} \frac{\dot{\alpha}}{\alpha}$ and $\frac{\dot{\alpha}_{s}}{\alpha_{s}}=\frac{\partial \ln F_{3}}{\partial \ln F_{1}} \frac{\dot{\alpha}}{\alpha}=\frac{\partial \ln F_{3}}{\partial \ln F_{2}} \frac{\partial \ln F_{2}}{\partial \ln F_{1}} \frac{\dot{\alpha}}{\alpha}$. Expecting the ratios $\left|\frac{\partial \ln F_{2}}{\partial \ln F_{1}}\right|$ and $\left|\frac{\partial \ln F_{3}}{\partial \ln F_{2}}\right|$ of the order unity, the three rates $\left|\frac{\dot{\alpha}_{w}}{\alpha_{w}}\right|,\left|\frac{\dot{\alpha}_{s}}{\alpha_{s}}\right|$ and $\left|\frac{\dot{\alpha}}{\alpha}\right|$ should be comparable, both at BBN and at the epoch of the Oklo phenomenon (see Uzan 2002).

We conclude that our modified Kaluza-Klein type action provides a good effective description of interactions at low energy. The instability problem (Paper I) is cured by the introduction of an additional external bulk scalar field minimally coupled to gravity. It accounts naturally for a cosmological time variation of $\alpha$, in agreement with recent data. It also reconcile the discordant laboratory measurements of $G$, by interpreting their differences as due to a coupling with the dipolar magnetic field of the Earth (Paper II).

Compared to other explanations, our assumption appears as an economical extension of general relativity. Like in the Barrow-Sandvik-Magueijo (BSM) work (Barrow et al. 2001), the variation of the effective fine structure constant is related to the coupling of a scalar field to the Maxwell invariant $F^{\alpha \beta} F_{\alpha \beta}$. Likewise, the effective fine structure constant remains constant during the radiation era or the future cosmological constant era. Further, BSM find that the product $G_{\text {eff }} \alpha_{\text {eff }}$ approaches an asymptotic constant in these era. In this paper, each of the two effective constant approaches its true value (and thus also the product). In addition, the quotient $G_{\text {eff }}^{3} / \alpha_{\text {eff }}$ remains approximately constant during the whole cosmic evolution, since $G_{\text {eff }}$ and $\alpha_{\text {eff }}$ vary respectively as $\Phi^{-1}$ and approximately $\Phi^{-3}$. Above all, the velocity of light in vacuum remains constant, as well as the electric charges. Moreover, our model derives from a very simple geometrical hypothesis. The first scalar field $\Phi$ (the fifteen degree of freedom of the metric) is purely geometric, and thus part of gravity in 5D. It affects directly the fine structure constant and the gravitational constant. In contrary to $\psi$, it is not minimally coupled to gravity (in 4D). Moreover, any kind of matter (except $\psi$ itself) acts as a source for the external scalar field $\psi$, and not only the EM field like in the BSM work.

The present model is limited and intends to be effective only. More precise predictions would result from a fundamental theory, in the same spirit.

\section{References}

Ahluwalia, D. V., \& Kirchbach, M. 2001, Int. J. Mod. Phys. D, 10, 811 Avelino, P. P., Martins, C. J. A. P., Rocha, G., \& Viana, P. 2000, Phys. Rev. D, 62, 123508

Avelino, P. P., Esposito, S., Mangano, G., et al. 2001, Phys. Rev. D, 64, 103505

Barrow, J. D., Sandvik, H. B., \& Magueijo, J. M. 2002a, Phys. Rev. $\mathrm{D}, 65,063504$

Barrow, J. D., Magueijo, J. M., \& Sandvik, H. B. 2002b, Phys. Lett. B, 541, 201

Battye, R. A., Crittenden, R., \& Weller, J. 2001, Phys. Rev. D, 63, 043505

Bekenstein, J. D. 1982, Phys. Rev. D, 25, 1527

Damour, T., \& Dyson, F. 1996, Nucl. Phys. B, 480, 37

Dvali, G., \& Zaldarriaga, M. 2002, Phys. Rev. Lett., 88, 091303

Fujii, Y., Iwamoto, A., Fukahori, T., et al. 2000, Nucl. Phys. B, 573, 377

Fujii, Y. 2002 [astro-ph/0204069]

Fujii, Y., Iwamoto, A., Fukahori, T., et al. 2002 [hep-ph/0205206]

Landau, L. D., \& Lifshitz, E. M. 1959, The Classical Theory of Fields (Addison-Wesley Publishing, Reading/Massachusetts), 289-293

Lichnerowicz, A. 1955, Théories relativistes de la gravitation et de l'électromagnétisme (Masson et Cie, Paris/France), 201-206 195, 349

Mbelek, J.-P., \& Lachièze-Rey, M. 2001 [gr-qc/0012086] (Paper I) Mbelek, J.-P., \& Lachièze-Rey, M. 2002 [gr-qc/0204064] (Paper II) Murphy, M. T., Webb, J. K., Flambaum, V. V., et al. 2001a, MNRAS, 327,1208

Murphy, M. T., Webb, J. K., Flambaum, V. V., et al. 2001b, MNRAS, 327,1223

Murphy, M. T., Webb, J. K., Flambaum, V. V., et al. 2001c, MNRAS, 327,1237

Murphy, M. T., Webb, J. K., Flambaum, V. V., et al. 2001d, MNRAS, 327,1244

Olive, K. A., \& Pospelov, M. 2002, Phys. Rev. D, 65, 08504

Salam, A., \& Strathdee, J. 1982, Ann. Phys., 141, 316

Sandvik, H. B., Barrow, J. D., \& Magueijo, J. M. 2002, Phys. Rev. Lett., 88, 031302

Sokolowski, M., \&. Golda, Z. A. 1987, Phys. Lett. B, 195, 349

Thiry, Y. 1948, CRASS, 226, 216

Uzan, J.-P. 2002 [hep-ph/0205340]

Webb, J. K., Murphy, M. T., Flambaum, V. V., et al. 2001, Phys. Rev. Lett., 87, 091301

Witten, E. 1982, Nucl. Phys. B, 195, 481 